\title{
Mapping Global Population Changes
}

\author{
Yujie Liu and Jie Chen
}

\section{Introduction}

The increase in greenhouse gas emissions caused by human activities is considered as the main cause of global warming (Stocker et al. 2013). The rapid growth of population and economic activities in the twentieth century has brought unprecedented pressure on climate and the environment, and population has become an important topic in climate change research (Min et al. 2011; Diaz and Moore 2017; Forzieri et al. 2017). Accurate and robust predictions of population size and spatial distribution will help to assess the impact of climate change on socioeconomic development, human health, and resource demand and distribution, and provide a scientific basis for designing strategies to control greenhouse gas emissions and developing mitigation and adaptation policies (Lutz and Kc 2011; Field et al. 2014; Gerland et al. 2014).

Climate scenarios constitute the basis of climate change research, and the rational setting of socioeconomic development scenarios is the core of climate change impact assessment (Van Vuuren et al. 2012). Shared socioeconomic pathways (SSPs) are reference pathways that describe alternative trends that may emerge in social and economic system development in the twenty-first century in the absence of

\footnotetext{
Authors: Yujie Liu, Jie Chen.

Map Designers: Yuanyuan Jing, Jing'ai Wang, Ying Wang. Language Editor: Yujie Liu.
}

Y. Liu $(\bowtie) \cdot$ J. Chen

Key Laboratory of Land Surface Pattern and Simulation, Institute of Geographic Sciences and Natural Resources Research, Chinese Academy of Sciences, Beijing, 100101, China

e-mail: liuyujie@igsnrr.ac.cn

J. Chen

University of Chinese Academy of Sciences, Beijing, 100049,

China

(C) The Author(s) 2022

P. Shi, Atlas of Global Change Risk of Population and Economic Systems, IHDP/Future Earth-Integrated

Risk Governance Project Series, https://doi.org/10.1007/978-981-16-6691-9_5

\section{Method}

climate change or climate policies (O'Neill et al. 2014). Five such SSP schemes (SSP1-5) have been developed. In particular, SSP1 is a sustainable solution that advances in technology and reduces reliance on carbon energy; SSP2 describes a medium-sized solution that can maintain the current trend and gradually reduce the reliance on carbon energy; and SSP3 is a regionalization program that leads to reduced trade flows, adverse institutional development, and poor adaptation to climate change. Based on these SSPs, preliminary studies have been conducted on demographic and economic changes in more than 150 countries (O'Neill et al. 2014). It is expected that there will be more attention paid to the size, as well as the spatial distribution of future populations in the SSP scenarios, with the increasing demand for demographic analysis in small areas related to climate change (Chi 2009; Raymer et al. 2012).

This study aims to evaluate the changes in the global population under SSP1-2030, SSP1-2050, SSP2-2030, SSP2-2050, SSP3-2030, and SSP3-2050. We produced the data at the global and regional scales. The spatial resolution of global projections is $0.5^{\circ} \times 0.5^{\circ}$, and the spatial resolution of regional projections is $0.25^{\circ} \times 0.25^{\circ}$. The four hotspot regions are the Bohai Rim area in China, the Qinghai-Tibet Plateau in China, the northeastern United States, and western Europe. The present results may provide a scientific basis for climate risk assessment and effective adaptations.

The methods for projecting population and its change for future SSP scenarios are as follows: (1) collecting the statistical data of national populations in 2005; (2) analyzing the projections of national populations under the three SSP 


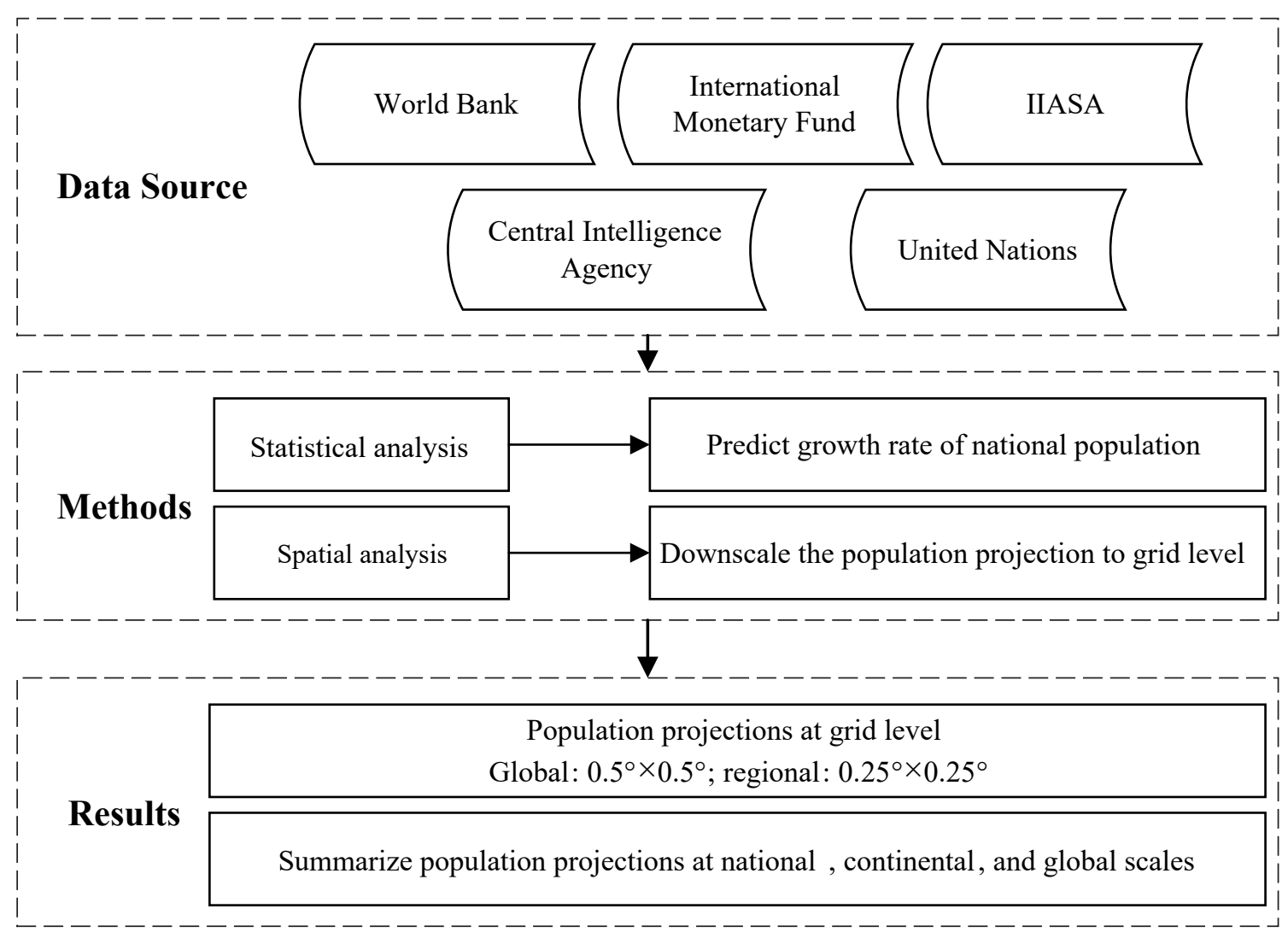

Fig. 1 Technical flowchart for the projection and mapping of the population of the world

scenarios in 2030 and 2050; (3) conducting the spatial downscaling of the populations at the country level to the grid level; and (4) summarizing the results of population projections. Figure 1 shows the technical flowchart for the projection and mapping of the populations of the world.

For population projections, the population at the country level in 2005 was obtained from the World Bank (https://data. worldbank.org.cn/), the International Monetary Fund (https:// www.imf.org/en/Data), the Central Intelligence Agency (https:// www.cia.gov/), and the United Nations (https://data.un.org/).

The global distribution of population in 2010 was assessed based on the population of the baseline period (2005) multiplied by the growth rate of population. The growth rate was calculated based on the population projections at the country level by the International Institute for Applied Systems Analysis (IIASA) (Samir and Lutz 2014). The statistical analysis was performed using ArcGIS. Then, the national population projections were spatially downscaled with reference to the spatial distribution of UN-WPP-Gridded Population of the World (GPW), v4 in 2005 (Doxsey-Whitfield et al. 2015). The spatial analysis was performed using ArcGIS. Then, the gridded population projections were summarized at the national, continental, and global scales, and the proportions of each continent to the world and the proportion of China were calculated for analysis.

\section{Results}

Global and continental populations under the SSP1-3 scenarios are shown in Fig. 2. The global population for the baseline period is $6575.50 \times 10^{6}$ persons, which increases to $9542.47 \times 10^{6}$ persons by 2050 under the SSP3 scenario. The increase in population is the largest for the SSP3 scenario and the smallest for SSP1. Among the continents, the population is the highest for Asia, followed by Africa, and the lowest in Oceania in both the baseline period (2010) and the SSP scenarios. Population in Asia and Africa in the baseline period is $61 \%$ and $15 \%$, respectively. The percentage of population under the three SSP scenarios decreases for Asia and increases for Africa. The percentage of the population by 2050 under the SSP3 scenario decreases for Asia to $57 \%$ and increases for Africa to $24 \%$ of the global total. Population in China is projected to increase under all the SSP scenarios and time periods compared to the baseline period. However, the percentage of the Chinese population in Asia and the world is likely to decrease. In the baseline period, China accounted for $33 \%$ and $20 \%$ of the population in Asia and the world, while in 2050 under the SSP3 scenario, the percentage would decrease to $24 \%$ and $14 \%$, respectively (Fig. 2). 


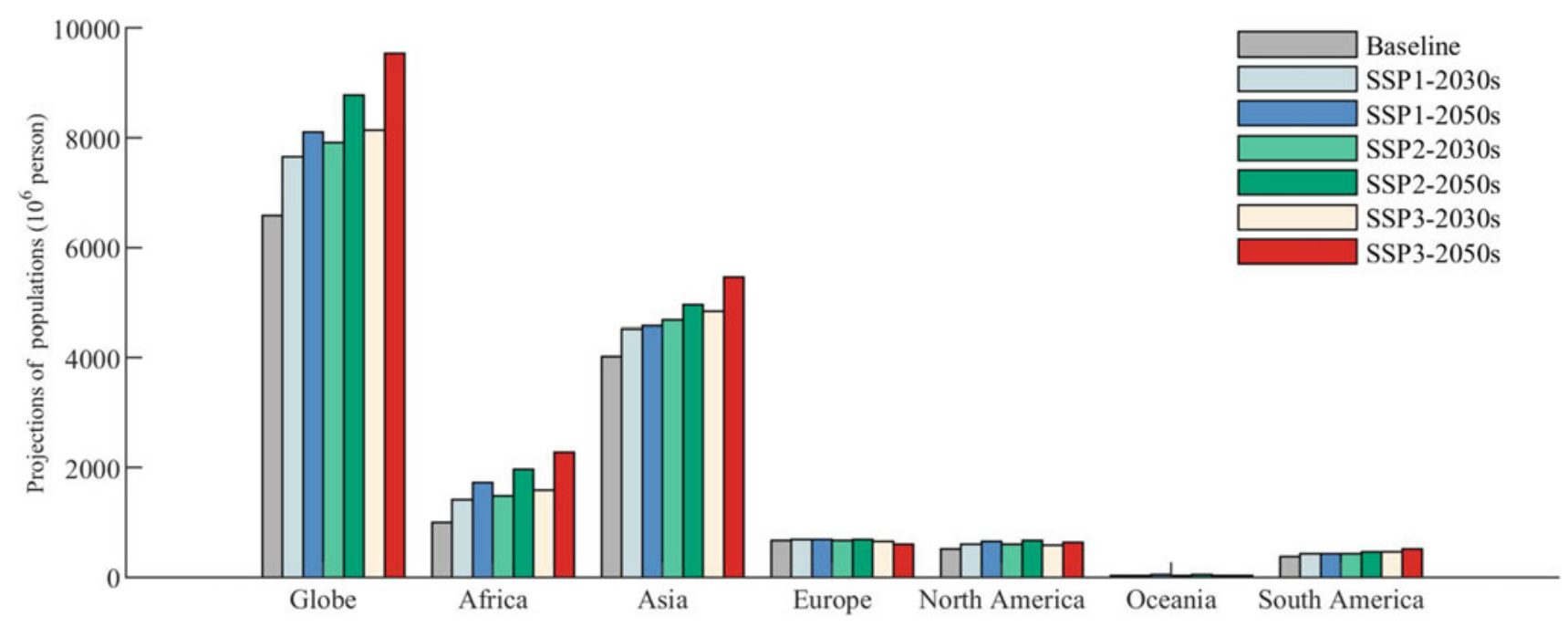

Fig. 2 Projections of global and continental populations under the Shared Socioeconomic Pathways 


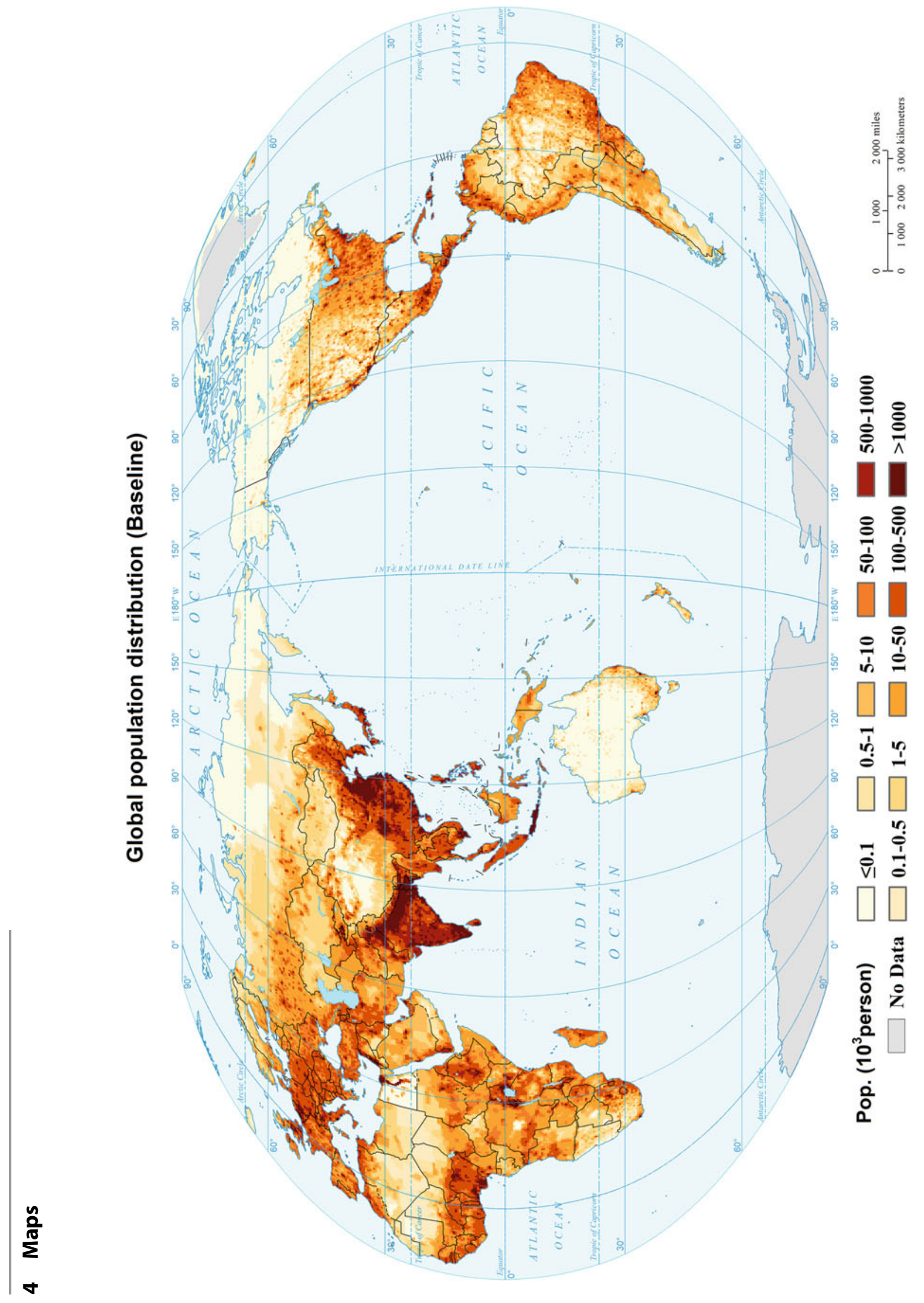




\section{Projected global population distribution (2030, SSP1)}

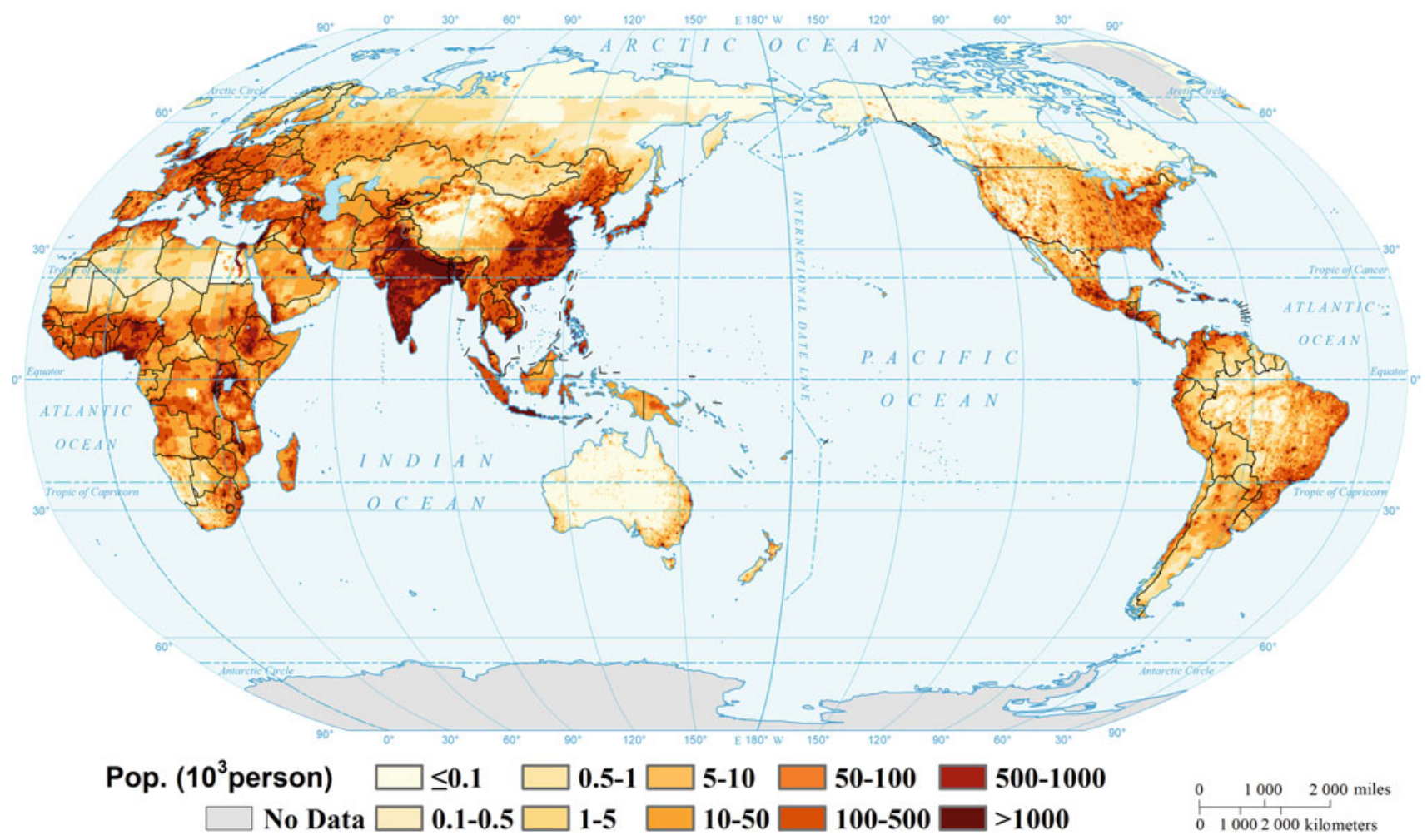

Projected global population distribution (2030, SSP2)

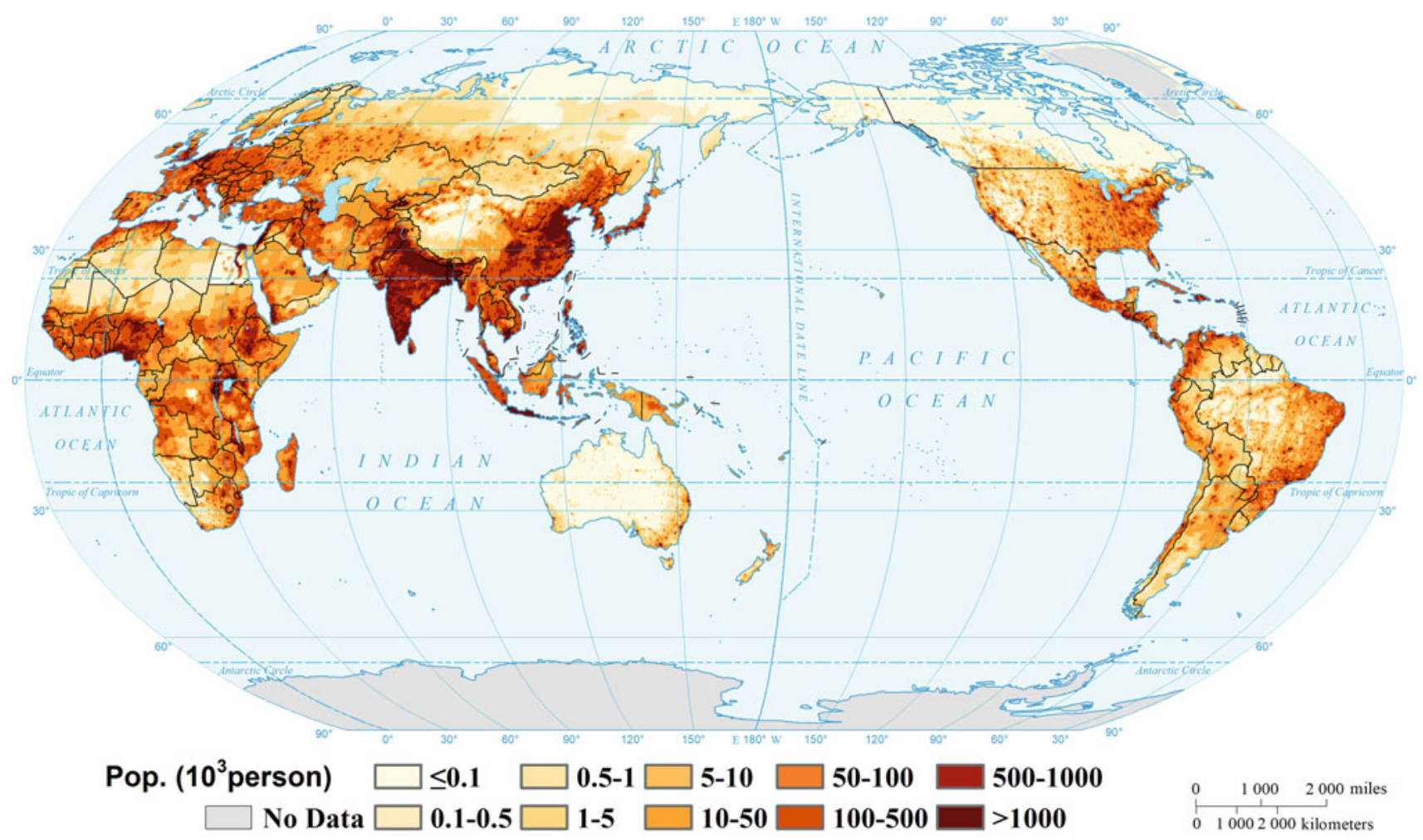




\section{Projected global population distribution (2030, SSP3)}

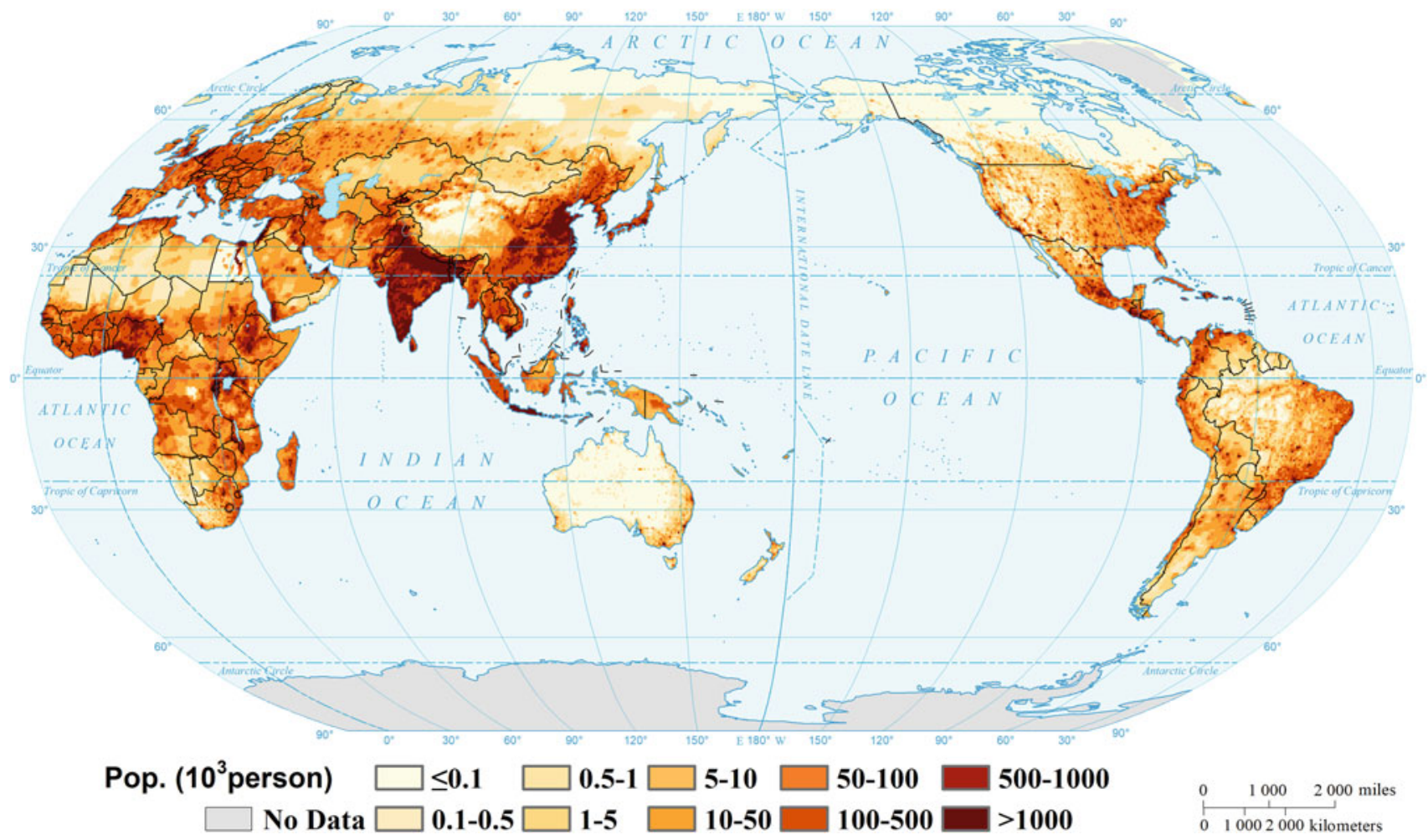

Projected global population distribution (2050, SSP1)

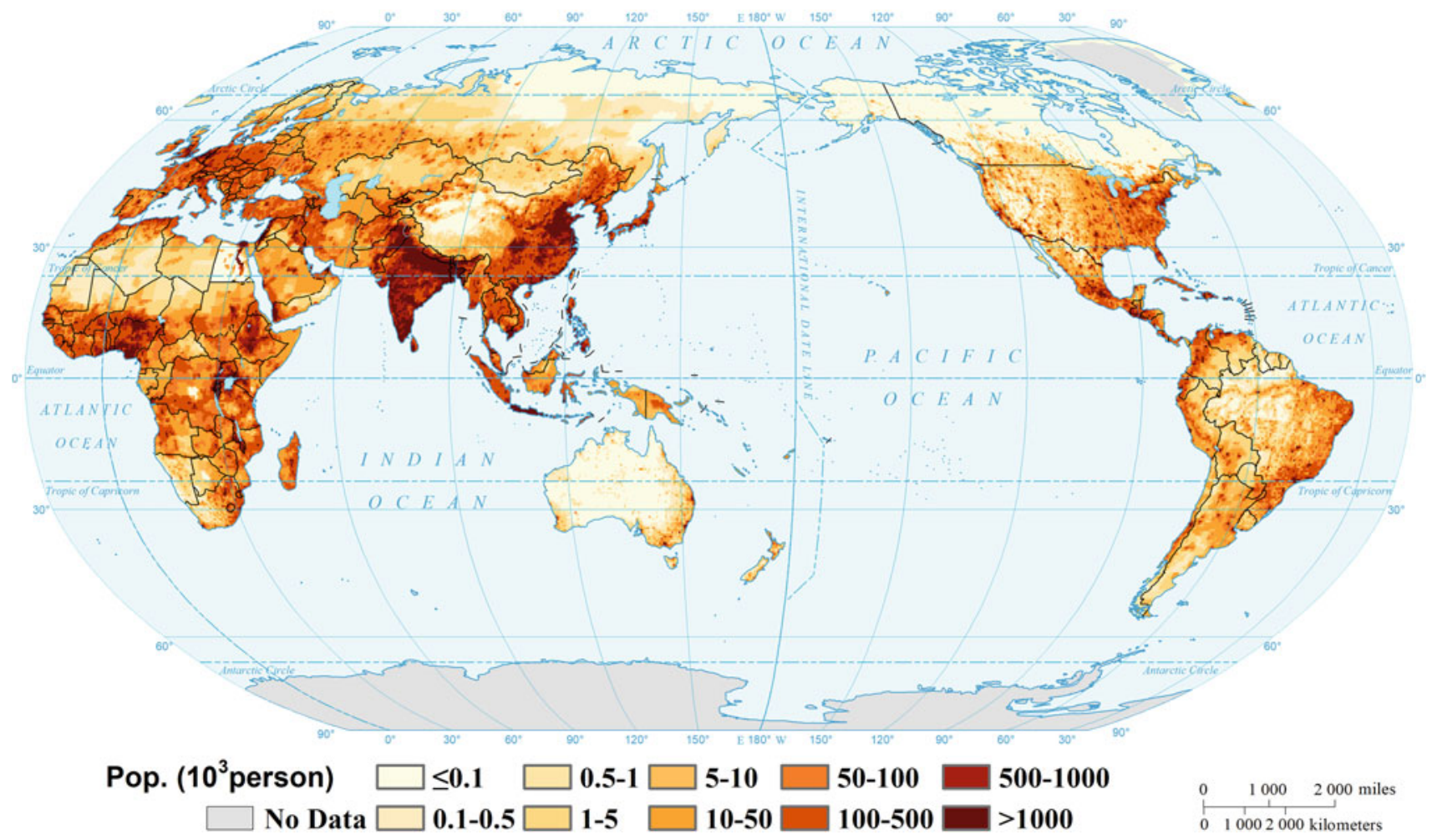




\section{Projected global population distribution (2050, SSP2)}

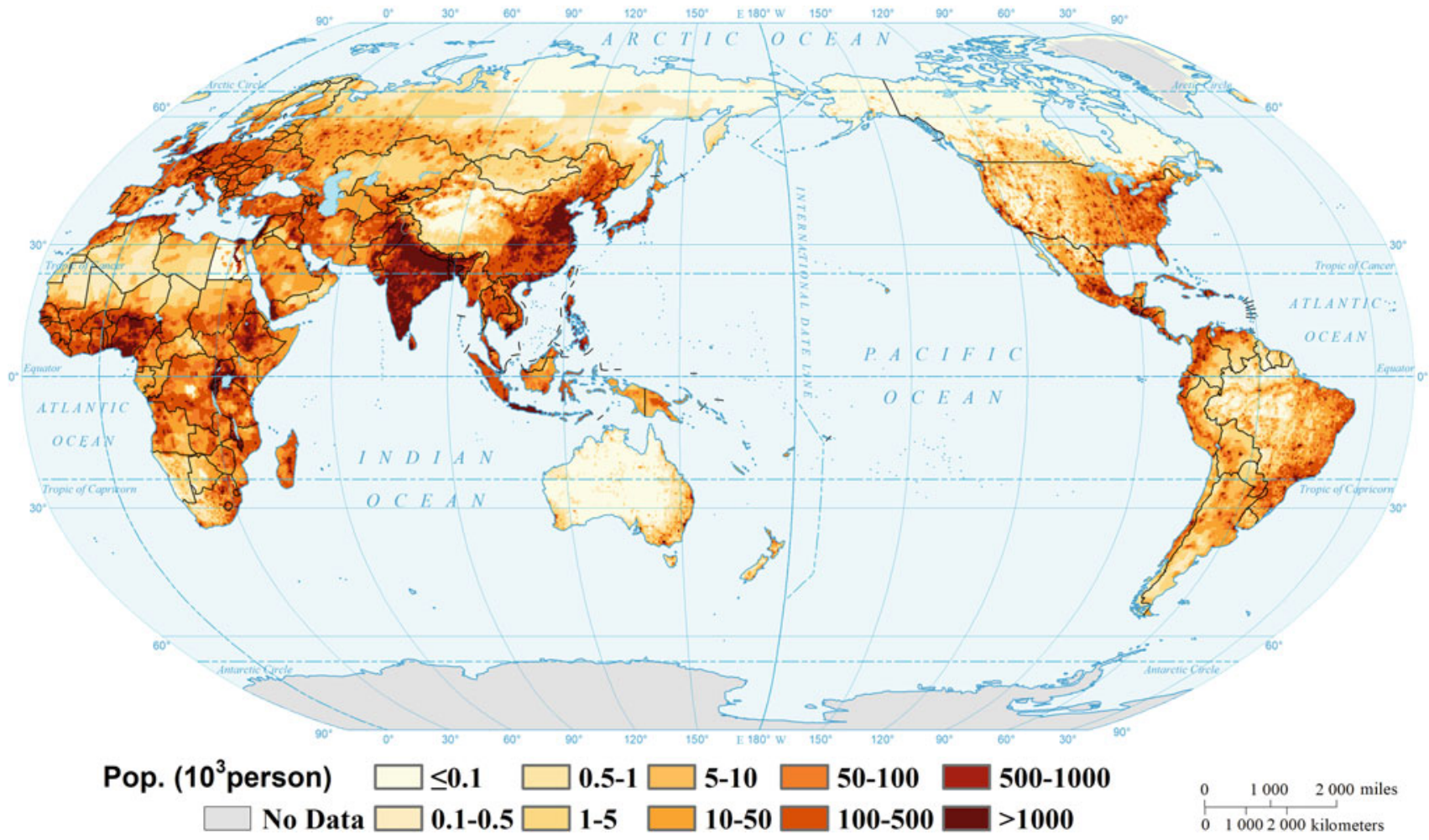

Projected global population distribution (2050, SSP3)

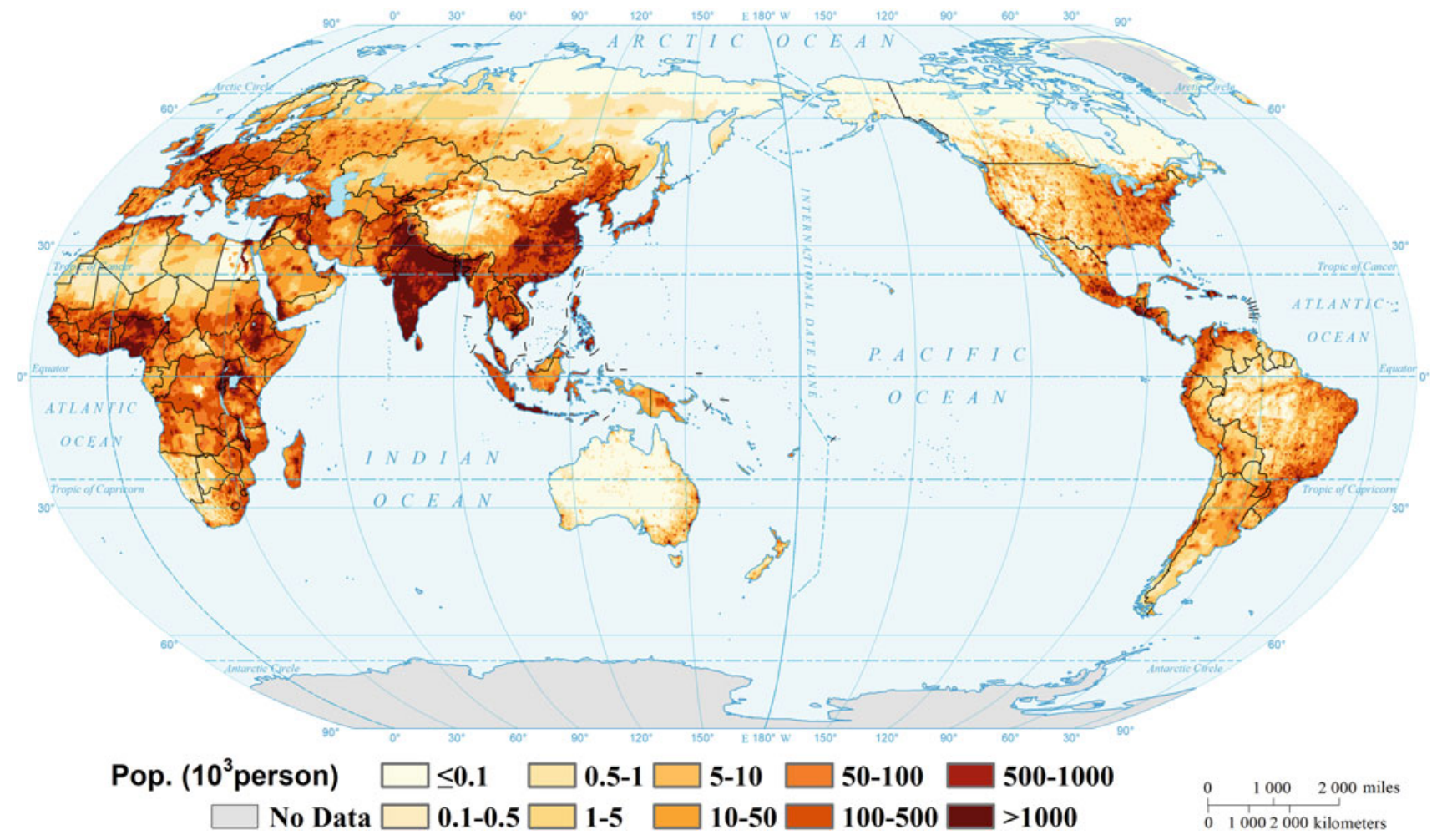




\section{References}

Chi, G. 2009. Can knowledge improve population forecasts at subcounty levels? Demography 46 (2): 405-427.

Diaz, D., and F. Moore. 2017. Quantifying the economic risks of climate change. Nature Climate Change 7 (11): 774-782.

Doxsey-Whitfield, E., K. MacManus, S.B. Adamo, L. Pistolesi, J. Squires, O. Borkovska, and S.R. Baptista. 2015. Taking advantage of the improved availability of census data: A first look at the gridded population of the world, version 4. Papers in Applied Geography 1 (3): 226-234.

Field, C.B., V.R. Barros, D.J. Dokken, K.J. Mach, M.D. Mastrandrea, T.E. Bilir, M. Chatterjee, K.L. Ebi, et al. 2014. Climate change 2014: Impacts, adaptation, and vulnerability. Part A: Global and sectoral aspects. In Contribution of working group II to the fifth assessment report of the intergovernmental panel on climate change. Cambridge, UK: Cambridge University Press.

Forzieri, G., A. Cescatti, F.B. Silva, and L. Feyen. 2017. Increasing risk over time of weather-related hazards to the European population: A data-driven prognostic study. The Lancet Planetary Health 1 (5): e200-e208.

Gerland, P., A.E. Raftery, H. Evikova, N. Li, D. Gu, T. Spoorenberg, L. Alkema, B.K. Fosdick, et al. 2014. World population stabilization unlikely this century. Science 346 (6206): 234-237.

Open Access This chapter is licensed under the terms of the Creative Commons Attribution 4.0 International License (http:// creativecommons.org/licenses/by/4.0/), which permits use, sharing, adaptation, distribution and reproduction in any medium or format, as long as you give appropriate credit to the original author(s) and the source, provide a link to the Creative Commons license and indicate if changes were made.
Lutz, W., and S. Kc. 2011. Global human capital: Integrating education and population. Science 333 (6042): 587-592.

Min, S.K., X. Zhang, F.W. Zwiers, and G.C. Hegerl. 2011. Human contribution to more-intense precipitation extremes. Nature 470 (7334): 378-381.

O’Neill, B.C., E. Kriegler, K. Riahi, K.L. Ebi, S. Hallegatte, T.R. Carter, R. Mathur, and D.P. van Vuuren. 2014. A new scenario framework for climate change research: The concept of shared socioeconomic pathways. Climatic Change 122 (3): 387-400.

Raymer, J., G.J. Abel, and A. Rogers. 2012. Does specification matter? Experiments with simple multiregional probabilistic population projections. Environment \& Planning 44 (11): 2664-2686.

Samir, K.C., and W. Lutz. 2014. Demographic scenarios by age, sex and education corresponding to the SSP narratives. Population and Environment 35 (3): 243-260.

Stocker, R.F., D. Qin, G.K. Plattner, M. Tignor, S.D. Allen, J. Boschung, A. Nauels, Y. Xia, et al. 2013. Climate change 2013: The physical science basis. Cambridge, UK: Cambridge University Press.

Van Vuuren, D.P., K. Riahi, R. Moss, J. Edmonds, A. Thomson, N. Nakicenovic, T. Kram, F. Berkhout, et al. 2012. A proposal for a new scenario framework to support research and assessment in different climate research communities. Global Environmental Change 22 (1): 21-35.
The images or other third party material in this chapter are included in the chapter's Creative Commons license, unless indicated otherwise in a credit line to the material. If material is not included in the chapter's Creative Commons license and your intended use is not permitted by statutory regulation or exceeds the permitted use, you will need to obtain permission directly from the copyright holder. 